\title{
BMJ Open Strength Training for Adolescents with cerebral palsy (STAR): study protocol of a randomised controlled trial to determine the feasibility, acceptability and efficacy of resistance training for adolescents with cerebral palsy
}

\author{
Jennifer M Ryan, ${ }^{1}$ Nicola Theis, ${ }^{2}$ Cherry Kilbride, ${ }^{1}$ Vasilios Baltzopoulos, ${ }^{1}$ \\ Charlie Waugh, ${ }^{3}$ Adam Shortland, ${ }^{4}$ Grace Lavelle, ${ }^{1}$ Marika Noorkoiv, ${ }^{1}$ \\ Wendy Levin, ${ }^{5}$ Thomas Korff ${ }^{1}$
}

To cite: Ryan JM, Theis N, Kilbride C, et al. Strength Training for Adolescents with cerebral palsy (STAR): study protocol of a randomised controlled trial to determine the feasibility, acceptability and efficacy of resistance training for adolescents with cerebral palsy. BMJ Open 2016;6: 012839 .

doi:10.1136/bmjopen-2016012839

\section{- Prepublication history} and additional material is available. To view please visit the journal (http://dx.doi.org/ 10.1136/bmjopen-2016012839).

Received 27 May 2016 Revised 1 September 2016 Accepted 9 September 2016

CrossMark

For numbered affiliations see end of article.

Correspondence to Dr Jennifer Ryan; Jennifer.ryan@brunel.ac.uk

\section{ABSTRACT}

Introduction: Gait is inefficient in children with cerebral palsy, particularly as they transition to adolescence. Gait inefficiency may be associated with declines in gross motor function and participation among adolescents with cerebral palsy. Resistance training may improve gait efficiency through a number of biomechanical and neural mechanisms. The aim of the Strength Training for Adolescents with cerebral palsy (STAR) trial is to evaluate the effect of resistance training on gait efficiency, activity and participation in adolescents with cerebral palsy. We also aim to determine the biomechanical and neural adaptations that occur following resistance training and evaluate the feasibility and acceptability of such an intervention for adolescents with cerebral palsy.

Methods and analysis: 60 adolescents (Gross Motor Function Classification System level I-III) will be randomised to a 10-week resistance training group or a usual care control group according to a computergenerated random schedule. The primary outcome is gait efficiency. Secondary outcomes are habitual physical activity, participation, muscle-tendon mechanics and gross motor function. General linear models will be used to evaluate differences in continuous data between the resistance training and usual care groups at 10 and 22 weeks, respectively. A process evaluation will be conducted alongside the intervention. Fidelity of the resistance training programme to trial protocol will be quantified by observations of exercise sessions. Semistructured interviews will be conducted with participants and physiotherapists following the resistance training programme to determine feasibility and acceptability of the programme.

Ethics and dissemination: This trial has ethical approval from Brunel University London's Department of Clinical Sciences' Research Ethics Committee and the National Research Ethics Service (NRES) Committee London-Surrey Borders. The results of the trial will be submitted for publication in academic journals, presented at conferences and distributed to
Strengths and limitations of this study

- The results of this trial will contribute to our understanding of the effect of resistance training on muscle-tendon mechanics in adolescents with cerebral palsy (CP), therefore bridging the gap between basic science and clinical efficacy.

- The findings of the process evaluation will improve the normalisation of the intervention into routine clinical practice by identifying individual, societal and contextual barriers to successful implementation of resistance training among adolescents with $\mathrm{CP}$.

- This is an efficacy study and therefore the results should not be considered conclusive.

adolescents, families and healthcare professionals through the media with the assistance of the STAR advisory group.

Trial registration number: ISRCTN90378161;

Pre-results.

\section{INTRODUCTION}

Cerebral palsy (CP) is a neurodevelopmental condition that begins in childhood and has a life-long impact on the individual and healthcare system. Although adults with mild-to-moderate CP have a relatively normal life expectancy, ${ }^{1}$ many experience deterioration in walking function between 20 and 40 years of age..$^{2-4}$ This can lead to physical inactivity, ${ }^{5} 6$ which in turn may result in increased risk of developing cardiovascular disease, ${ }^{5}{ }^{7-9}$ and limit participation and social integration in the home and community. The oxygen cost of walking, which is 
often used as a measure of gait efficiency, ${ }^{10}$ is high among children and adolescents with $\mathrm{CP}$ in comparison to their peers with typical development. ${ }^{11-13}$ Gait inefficiency may contribute to the negative cycle of physical inactivity, deterioration in walking function and physical de-conditioning. ${ }^{14}$ Reduced gait efficiency is also negatively associated with gross motor function and participation in mobility-related activities, ${ }^{15}$ and may cause fatigue, which further exacerbates deterioration in walking function. ${ }^{3}$ As gait is most inefficient at age 12 years in $\mathrm{CP}$ and gross motor function skills deteriorate shortly afterwards, ${ }^{16}$ targeted interventions during adolescence could disrupt this negative cycle.

Resistance training, which is commonly used for people with CP, has the potential to improve gait efficiency through a number of biomechanical and neural mechanisms. First, co-contraction of antagonistic muscles is exacerbated in $\mathrm{CP}^{17-19}$ and contributes to gait inefficiency. ${ }^{20}$ Studies involving children with typical development indicate that early resistance training-induced strength gains are predominantly neural in nature. ${ }^{21-23} \mathrm{~A}$ study of children with $\mathrm{CP}$ suggests that a reduction in antagonistic co-contraction may be a partial contributor. ${ }^{24}$ Second, excessive lengthening of the muscle fascicles during the single stance phase of walking could contribute to the increased energy cost of walking among people with CP. In typically developed gait it has been shown that in spite of significant length changes of the plantarflexor muscle-tendon unit as a whole, triceps surae muscle fascicles operate almost isometrically during the early to mid-stance phase of walking. ${ }^{25}$ In the subsequent propulsive phase, the fascicles shorten at velocities associated with high efficiency. ${ }^{26-28}$ This has been proposed to be an energy-saving mechanism during walking. ${ }^{29}$ Pilot data suggest that in children with $\mathrm{CP}$ and equinus gait, the fascicles of the gastrocnemius muscle lengthen during the single support phase of walking, ${ }^{30} 31$ rather than act isometrically, potentially due to the muscle's inability to resist the tensile forces in stance. Since eccentric muscle contractions are metabolically less efficient than isometric contractions, ${ }^{32}$ this may contribute to walking inefficiency in people with CP. An increase in the volume and strength of these muscles due to resistance training may have a positive effect on oxygen cost by enabling fascicles to work more isometrically during gait. Finally, the degree to which the muscle shortens during dynamic contractions is governed by the elastic properties of muscles and tendons (ie, stiffness). It has been previously demonstrated that children with CP have an increased passive stiffness and disproportionally long Achilles tendon. ${ }^{33} 34$ This may reduce the ability of the muscle to generate adequate force in the propulsive phase of gait, as fascicles are not operating at optimum lengths or velocities. Resistance training has been shown to increase tendon stiffness by up to $25 \%$ in children with typical development, ${ }^{35}$ thus demonstrating the potential to affect the ratio of muscle to tendon stiffness in CP in a way that promotes a more efficient muscle-tendon interaction and muscle contraction during gait. Such changes may improve gait efficiency in this population.

Resistance training has been advocated for adolescents with CP for decades as it may delay the loss of mobility in adulthood by increasing muscular reserve ${ }^{36}$ reduce the risk of chronic disease such as type II diabetes mellitus, ${ }^{37} 38$ and improve walking function. ${ }^{39}{ }^{40}$ However, current evidence for the use of resistance training to improve walking function is equivocal ${ }^{41-43}$ and thus, clarity is required. The uncertainty regarding the effects of resistance training on walking function may be due to a number of shortcomings in current research. First, many resistance training protocols are of an inadequate volume, intensity or duration. ${ }^{38}$ For neural and musculotendinous adaptations to occur, an adequate training stimulus and duration are required. ${ }^{44}$ In addition, multijoint exercises are often used in resistance training programmes. As people with CP have severe muscle weakness, they may compensate with stronger muscles when performing multijoint exercises. ${ }^{39} 40$ The efficacy of resistance training may also vary according to the muscle group targeted. Resistance training in $\mathrm{CP}$ is primarily targeted at the knee extensors ${ }^{39} 4345$ despite muscle deficits being most pronounced at the ankle. ${ }^{46} 47$ Plantarflexor strength is associated with walking function and step cadence in $\mathrm{CP} ;{ }^{48}{ }^{49}$ and pilot data suggest that progressive resistance training of the plantarflexors using single-joint exercises improves muscle strength, muscle volume, ${ }^{50}$ walking speed and stride length. ${ }^{51}$ Further, the underlying biomechanical and neural adaptations to resistance training are poorly understood in people with CP. When evaluating a complex intervention, it is imperative that a theoretical understanding of the likely process of change is developed. ${ }^{52}$ The paucity of research into resistance training-induced adaptations in $\mathrm{CP}$ means that current clinical practice is potentially based on erroneous assumptions.

We hypothesise that training the ankle plantarflexors with progressive resistance training will result in increases in gait efficiency through the biomechanical and neural adaptations outlined above. The aims of the Strength Training for Adolescents with CP (STAR) trial are to (1) evaluate the effect of resistance training of the ankle plantarflexors on gait efficiency, activity and participation in adolescents with CP, (2) determine the biomechanical and neural adaptations that occur following resistance training in adolescents with $\mathrm{CP}$ and (3) evaluate the feasibility and acceptability of resistance training for adolescents with CP.

\section{METHODS AND ANALYSIS}

\section{Study design}

This is a multicentre randomised controlled trial with a parallel process evaluation (figure 1). Participants will be recruited through the National Health Service, schools and organisations for people with disabilities 


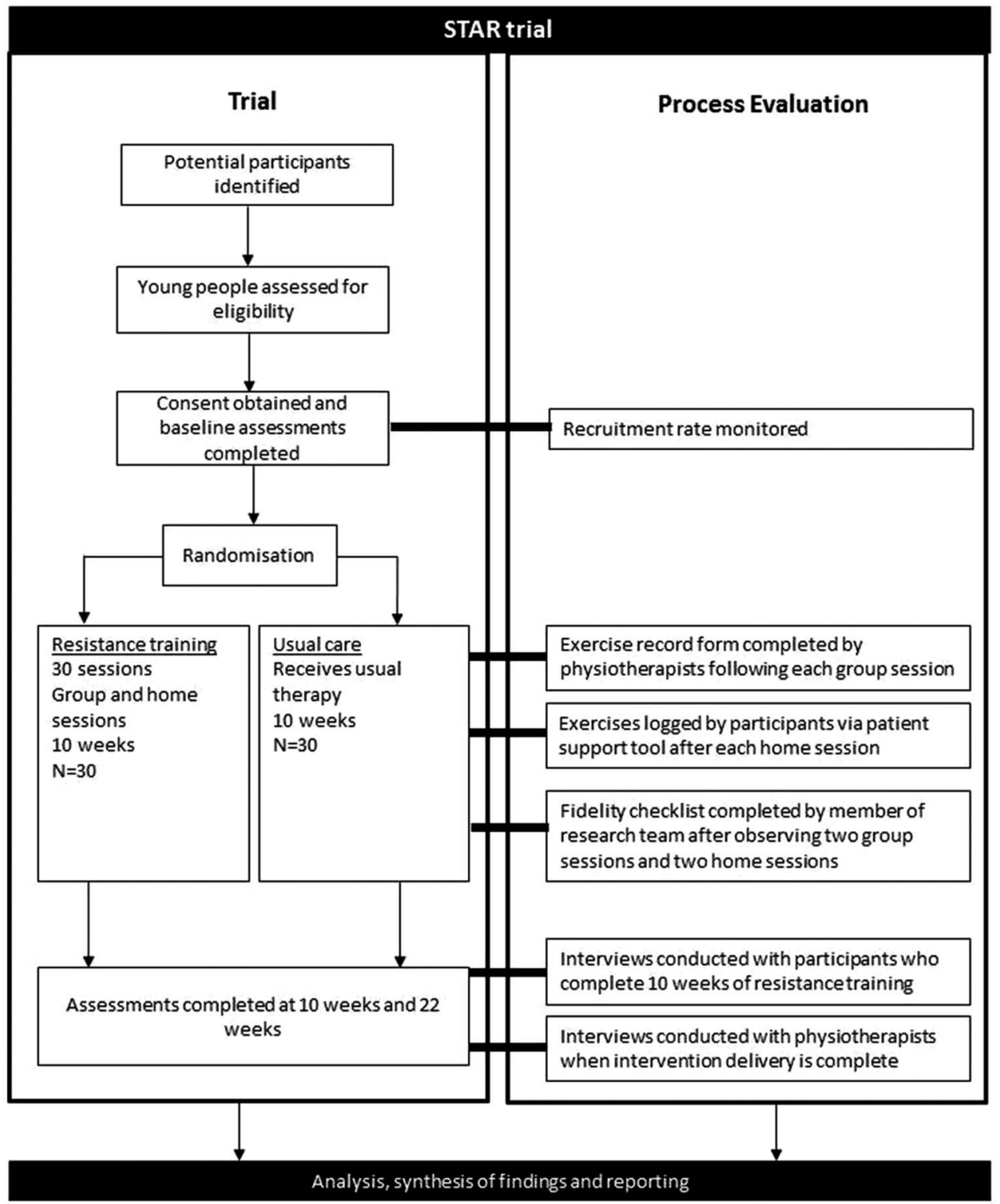

Figure 1 Experimental design for the STAR trial. STAR, Strength Training for Adolescents with cerebral palsy.

throughout England. Assessments will be conducted at Brunel University London. Participants will be randomly assigned to one of two groups (exercise or usual care control group) in a 1:1 ratio after completing baseline assessments. Allocation will be performed by an individual independent to the study according to a computergenerated random schedule in permuted blocks of two or four within Gross Motor Function Classification System (GMFCS) level strata (levels I-II and level III). The allocation of participants to each group within each stratum will be placed in sequentially numbered, opaque, sealed envelopes, so that participants and researchers enrolling participants cannot foresee assignment. All participants will be instructed to continue their usual physiotherapy and activities, provided it does not include progressive resistance training. Usual physiotherapy and participation in physical activities will be monitored at baseline, 10 and 22 weeks. A usual care checklist, developed from the results of a national survey of physiotherapists in the UK regarding usual care for adolescents with $\mathrm{CP}$, will be used to monitor usual physiotherapy.

\section{Trial status}

At the time of submission of this study protocol, the trial is ongoing and recruiting participants. Recruitment began in August 2015 and is expected to end in August 2017.

\section{Participants}

Sixty adolescents will be recruited. The inclusion criteria for the proposed trial are (1) adolescents with spastic CP aged 10-19 years (amended from 12 to 19 years on 15 February 2016), (2) the ability to walk independently 
with or without a mobility aid (ie, GMFCS levels I-III) and (3) the ability to activate the ankle plantarflexors. Individuals in GMFCS level I are able to walk indoors and outdoors without assistance and can perform gross motor skills such as running and jumping, but speed, balance and coordination are reduced. Those in level II can also walk indoors and outdoors without assistance, but have only minimal ability to perform gross motor skills like running and jumping. Individuals in level III require a mobility aid to walk independently and may use wheeled mobility to travel long distances. ${ }^{53}$ Participants' ability to activate their plantarflexors on the right and left leg will be assessed using a modified version of the Selective Control Assessment of the Lower Extremity (SCALE) ${ }^{54}$ Participants will be asked to dorsiflex, plantarflex and dorsiflex the ankle, while sitting with their legs over the edge of the examination table with the knee extended as much as possible, and the calf supported by the assessor. This assessment will be performed by the person identifying eligible participants where possible and also by the research team when the participant attends the baseline assessment. Participants' ability to activate their plantarflexors will be rated as 'normal' if they can achieve $15^{\circ}$ of active plantarflexion in the sagittal plane, 'impaired' if they achieve $<15^{\circ}$ active plantarflexion in the sagittal plane or if plantarflexion is accompanied by ankle inversion, 'no active movement' if they do not achieve active plantarflexion at the ankle but the plantarflexors are activated as determined by palpation, and 'unable' if the plantarflexors are not activated as determined by palpation. Adolescents who are unable to activate their plantarflexors will be excluded from participating in the study.

Exclusion criteria are (1) orthopaedic surgery of the lower extremities in the past 12 months, (2) botulinum toxin type A (Botox) injections in the past 6 months, (3) serial casting in the past 6 months and (4) insufficient cognitive understanding to comply with the assessment procedures and training programme.

\section{Resistance training intervention}

The progressive resistance training intervention is based on current guidelines for youth resistance training. ${ }^{44}$ Adolescents in the resistance training group will participate in 30 sessions of progressive resistance training over 10 weeks; 10 supervised group sessions will be carried out at participants' local physiotherapy department or gym and 20 sessions will be carried out at participants' homes. There will be at least 24 hours between each session. Participants will complete a physical activity readiness questionnaire prior to participation in resistance training. Single-joint plantarflexor exercises will be prescribed based on individual strength capacities to ensure the participant can perform a minimum of 6 repetitions and no more than 15 repetitions, that is, 6-15 repetition maximum (RM) which equates to $\sim 50-85 \%$ of $1 \mathrm{RM}$, in line with current guidelines. ${ }^{44}$ Each participant will be familiarised with the exercises and taught the correct technique prior to starting the programme. Participants will be provided with an OMNI perceived exertion scale at their first appointment to monitor perceived intensity. The physiotherapist delivering the programme will prescribe at least one of the following exercises each week depending on the adolescent's individual capacity: (1) seated straight knee calf press against resistance band (TheraBand, Hygenic Corporation), (2) seated straight knee calf press with a leg press machine, (3) standing calf raises against body weight with or without additional resistance and (4) standing calf raises in a hack squat machine (table 1). For all exercises, participants will be instructed to keep the knee joint as straight as possible to ensure activation of all triceps surae muscles ${ }^{5-57}$ and perform the exercise through full available range of ankle movement where possible.

A periodised progressive resistance training programme will be used to ensure continued adaptation throughout the 10 weeks (table 2). Exercises will be performed at moderate velocity, that is, $1-2 \mathrm{~s}$ concentric,

Table 1 Description of plantarflexor exercises performed by adolescents in intervention group

\begin{tabular}{|c|c|c|c|}
\hline Exercise & Starting position & $\begin{array}{l}\text { Resistance added in } \\
\text { group session }\end{array}$ & $\begin{array}{l}\text { Resistance added } \\
\text { in home session }\end{array}$ \\
\hline $\begin{array}{l}\text { Seated straight knee calf } \\
\text { press against resistance } \\
\text { band }\end{array}$ & $\begin{array}{l}\text { Long sitting with knee extended; ankle in } \\
\text { dorsiflexion; band placed around the ball of the } \\
\text { foot and both ends of the band held by the } \\
\text { participant }\end{array}$ & $\begin{array}{l}\text { Resistance band of } \\
\text { varying resistance }\end{array}$ & $\begin{array}{l}\text { Resistance band of } \\
\text { varying resistance }\end{array}$ \\
\hline $\begin{array}{l}\text { Standing straight knee } \\
\text { calf raise }\end{array}$ & $\begin{array}{l}\text { Standing with knee extended; ankle in } \\
\text { dorsiflexion; ball of foot on step }\end{array}$ & $\begin{array}{l}\text { Body weight/weighted } \\
\text { vest/ankle weights }\end{array}$ & $\begin{array}{l}\text { Body weight/ } \\
\text { weighted vest/ankle } \\
\text { weights }\end{array}$ \\
\hline $\begin{array}{l}\text { Standing straight knee } \\
\text { calf raise with hack squat } \\
\text { machine }\end{array}$ & $\begin{array}{l}\text { Standing in hack squat machine with knee } \\
\text { extended; ankle in dorsiflexion; foot flat on } \\
\text { footplate }\end{array}$ & $\begin{array}{l}\text { Free weights added } \\
\text { to hack squat } \\
\text { machine }\end{array}$ & $\begin{array}{l}\text { Not completed at } \\
\text { home }\end{array}$ \\
\hline $\begin{array}{l}\text { Seated straight knee calf } \\
\text { press with leg press } \\
\text { machine }\end{array}$ & $\begin{array}{l}\text { Sitting in leg press with knee extended; ankle in } \\
\text { dorsiflexion; foot on footplate }\end{array}$ & $\begin{array}{l}\text { Free weights added } \\
\text { to leg press machine }\end{array}$ & $\begin{array}{l}\text { Not completed at } \\
\text { home }\end{array}$ \\
\hline
\end{tabular}


Table 2 Periodised progression of resistance exercises performed by adolescents in intervention group

\begin{tabular}{lllll}
\hline Weeks & Sets & Repetitions & Intensity & Muscle action \\
\hline 1 and 2 & 4 & 12 & $12 \mathrm{RM}$ & ECC and CON \\
3 and 4 & 6 & 12 & $12 \mathrm{RM}$ & ECC and CON \\
5 and 6 & 6 & 8 & $8 \mathrm{RM}$ & ECC and CON \\
7 and 8 & 8 & 8 & $8 \mathrm{RM}$ & ECC and CON \\
9 and 10 & 8 & 6 & $6 \mathrm{RM}$ & ECC and CON
\end{tabular}

CON, concentric; ECC, eccentric; RM, repetition maximum.

1-2 s eccentric. A rest period of at least 2 minutes will be allowed between each set.

Load will be added to each prescribed exercise incrementally at every supervised session to ensure that the participant can complete the prescribed number of repetitions in each set to fatigue. For example, in week 8 , the participant's eight RM will be determined at the start of the supervised session for each prescribed exercise by adding load to the exercise, so that the participant performs eight repetitions to fatigue in each set. Fatigue will be indicated by participants' refusal to complete another repetition, an inability to perform another full repetition, or improper form that results in the plantarflexors not being activated. The load may be adjusted during the supervised session depending on the individual's capacity to ensure that they are reaching fatigue during each set.

For the seated straight knee calf press against resistance band, the band will be placed around the ball of the foot (see online supplementary file 1 ). The length of the resistance band and the position of the participants' hands when holding the band will be kept constant during this exercise. Participants will plantarflex at the ankle and push against the resistance band. When a participant can complete more than the prescribed number of repetitions, they will progress to a stiffer resistance band until they are able to progress to a different exercise. The stiffness of the resistance band increases according to the colour of the band, from least resistance to most resistance as follows: yellow, red, green, blue, black, silver, gold. For the standing calf raise exercise, participants will be instructed to raise and lower their body using their calf muscle. If necessary, they will be allowed to hold onto a chair for balance (see online supplementary file 2). Participants may also complete standing calf raises using a hack squat machine in the group session (see online supplementary file 3). For the seated straight knee calf press with the leg press machine, participants will be instructed to push against the footplate with the ball of their foot (see online supplementary file 4 ).

Participants will not perform all four exercises at each session. Instead, they will perform a combination of one or more exercises, depending on their individual strength capacity each week. Each participant will perform the prescribed exercise(s) until they complete the required number of sets and repetitions of plantarflexor exercises on each leg. For example, in week 1, a participant may be prescribed four sets of 12 repetitions of seated straight knee calf presses against a resistance band on each leg. In week 2, if they are able to perform $>12$ repetitions against the strongest resistance band they may be prescribed four sets of 12 repetitions of standing calf raises on each leg. They could also be prescribed two sets of 12 repetitions of standing calf raises and two sets of 12 repetitions of the seated straight knee calf press with the leg press machine, on each leg. The number and type of exercises prescribed will be at the discretion of the physiotherapist provided they adhere to the prescribed repetitions, sets and intensity for a given week.

Unilateral exercises will be performed where possible. If a participant is unable to perform unilateral exercises, they will perform bilateral exercises initially until they are able to progress to unilateral exercises.

Each supervised session will be managed by a physiotherapist who has received training in delivering the resistance training programme. All physiotherapists delivering the intervention will receive a standardised clinician manual and on-site training. Participants are advised to perform a 5-10 min aerobic warm-up of lower limb aerobic exercise and a 5 min cool-down consisting of lower limb aerobic exercise and plantarflexor stretches at each session. At the start of each session, the physiotherapist will ask participants if they experienced any adverse events since the last supervised session and record reported events. At the end of the class, the physiotherapist will ask participants if they experienced any adverse events during the class, which will also be recorded. At the end of the supervised session, the physiotherapist will outline the exercises each participant has to complete at home and provide any equipment required to complete the exercises. The type of exercise and amount of load added to the exercise will be based on the exercise(s) the participant performed in the supervised session. Participants will be provided with a paper exercise diary containing photographs of the prescribed exercises and the prescribed number of repetitions and sets for each exercise. They will also be provided with videos on how to perform their home exercises via a patient support tool (Salaso Health Solutions, Kerry, Ireland), which they will access through the website or the application on a smartphone or tablet.

\section{Outcome measures}

Participants in both groups will be tested for the following dependent variables at baseline, at 10 weeks (immediately postintervention), and at 22 weeks (12 weeks postintervention). Attempts will be made to collect all outcome data for participants who discontinue the intervention. 


\section{Primary outcome measure}

\section{Gait efficiency}

Gait efficiency will be measured by a blinded assessor using a protocol outlined by Schwartz. ${ }^{58}$ Participants' mass to the nearest $0.1 \mathrm{~kg}$ and stature to the nearest $0.1 \mathrm{~m}$ will be measured. Oxygen consumption will be recorded using a portable metabolic system (Cosmed $\mathrm{K} 5$, Rome, Italy) during a $10 \mathrm{~min}$ rest period in a semirecumbent position and during $6 \mathrm{~min}$ of overground walking at a self-selected speed. Participants' average walking speed will be measured in 1 min intervals. Prior to each test the Cosmed K5 will be calibrated for oxygen and carbon dioxide concentration and turbine flow according to the manufacturer's recommendations. Participants will be asked to fast for 4 hours prior to the test and will wear their usual footwear and orthotic devices, and use their usual assistive devices, if any.

Steady state breath will be determined using the protocol outlined by Schwartz. ${ }^{58}$ Net non-dimensional oxygen cost (NNcost) will be used to indicate gait efficiency as this has been shown to be the best measure of gait efficiency in children with $\mathrm{CP} .^{59} 60$

\section{Secondary outcome measures}

\section{Physical activity}

Physical activity will be measured using an accelerometer (Actigraph wGT3X) worn on the waist above the right hip or least affected side in the case of significant asymmetry, in the midaxillary line. Participants will be asked to wear the accelerometer for 7 consecutive days at each assessment point. Participants with at least 2 days of monitoring will be included in the analysis as 2 days of monitoring is necessary to achieve a reliability coefficient of 0.70 for adolescents with $\mathrm{CP}^{61}$ Time spent in sedentary, light, moderate-to-vigorous activity will be determined by classifying accelerometer counts according to cut-points validated in children and adolescents with CP. ${ }^{62}$ Time in sedentary behaviour as a percentage of total wear-time and mean accelerometer counts per minute will also be calculated.

\section{Participation}

Participation, defined as 'involvement in a life situation' by the WHO's International Classification of Functioning, Disability and Health (ICF), ${ }^{63}$ will be assessed using the Assessment of Life Habits (Life-H) short form questionnaire V.3.1. The Life-H short form questionnaire was developed to measure the quality of social participation in adolescents and adults by drawing information on the individual's own perception of their performance. It contains 77 items divided into 12 life habit and social role categories. The 12 categories include nutrition, fitness, personal care, communication, housing, mobility, responsibilities, interpersonal relationships, community life, education, employment and recreation. Each domain can be analysed in terms of level of accomplishment, type of assistance required and level of satisfaction. Level of accomplishment and level of satisfaction are scored on a five-point ordinal continuum, while level of assistance is attributed a score based on a four-point scale ranging from no assistance to human assistance. For scoring purposes, an accomplishment score is derived though combination and weighting of level of difficulty and types of assistance scores and computation of the following: $\left(\sum\right.$ scores $\left.\times 10\right) /$ (number of applicable life habits $\times 9$ ). A higher score is indicative of greater participation. The Life-H demonstrated content validity and adequate-to-good levels of test-retest reliability on initial tool development in adolescents and adults. ${ }^{64}$ It has also been successfully implemented in cohort studies examining participation levels of children and adolescents with CP. ${ }^{6566}$

\section{Muscle-tendon mechanics}

The following muscle-tendon mechanics will be assessed on the participant's affected leg in the case of unilateral CP and the most affected leg in the case of bilateral CP. If a participant reports that both sides are equally affected, the right leg will be assessed. Two researchers blinded to group allocation will conduct the assessments.

\section{Muscle strength}

Plantarflexor muscle strength will be assessed on an isokinetic dynamometer (Cybex). For this purpose, participants will be secured in a seated position with the knee joint as close to full extension as possible and the foot secured to the footplate of the dynamometer. The medial malleolus of the ankle will be aligned with the centre of rotation of the dynamometer arm. The dynamometer footplate will be positioned as close to perpendicular to the horizontal axis as possible, dependent on each participant's available joint range of motion. To reduce the risk of leg, upper body or heel movement during contractions, stabilisation straps will be applied tightly over the foot, thigh and torso, and participants will be instructed to cross their arms over their chest.

Each participant will be required to push against the footplate producing a maximal voluntary isometric contraction manoeuvre while plantarflexor torque is recorded. To familiarise the participants with the equipment and the plantarflexion task, three to five submaximal isometric contractions will be performed with the instruction to 'rotate the foot away from the body while keeping the heel in contact with footplate'. These contractions also provide a task-specific warm-up to precondition the tendon and ensure consistency of load-deformation properties. ${ }^{6768}$ After a mandatory 5 min rest period to minimise fatigue, participants will then perform three further contractions with maximal effort.

Antagonist muscle activity accompanies agonist muscle activation during maximal contractions, ${ }^{23} 69$ which contributes a dorsiflexion torque to the plantarflexion. As a result, the net joint torque measured by the dynamometer is an underestimation of the actual torque developed by the agonist muscle. To account for this 
co-activation, we will first measure tibialis anterior muscle activity using surface electromyography (EMG) (Delsys) during a slow, submaximal ramped isometric dorsiflexion trial, completed after the final plantarflexion contractions. Second, an EMG-torque relationship will be constructed and fitted with a polynomial function which will then be used to estimate the antagonist torque present during the plantarflexion trials from EMG amplitude. This torque will be used to quantify antagonistic co-contraction during the isometric maximal effort. It will then be added to the net joint torque measured by the dynamometer to yield the plantarflexor torque.

\section{Muscle anatomical cross-sectional area}

Three-dimensional (3D) freehand ultrasonography method will be used to measure muscle volumes, anatomical cross-sectional area (ACSA) and lengths of the medial and lateral gastrocnemii as well as the soleus muscle. ${ }^{70}$ Two-dimensional ultrasound images of the transverse plane of the muscle with 3D motion data will be collected using Stradwin software (V.5.1, Mechanical Engineering, Cambridge University, Cambridge, UK). Participants will be asked to lie prone on an examination table with the foot extended off the edge of the table for $10 \mathrm{~min}$ prior to the assessment of muscle size, to allow fluid shifts in the muscle to stabilise. ${ }^{71}$ The midpoint of the line connecting the lateral and medial tibial plateau and tuberositas calcanei will be marked on the skin and the distance between these points will be measured. The starting point of all ultrasound sweeps will be the line connecting the medial and lateral tibial plateau. The same starting point of the sweeps and the measured distance between the tibial plateau and calcanei will guide the standardisation of muscle length and CSA analysis. Participants will be instructed to relax their calf muscles during the imaging. A strap will be placed over the participant's thighs just above the knee and a researcher will apply a gentle pressure on the heel in order to avoid any movement of the limbs. Hip and knee joints will be fully extended, unless full joint extension is not possible, in which case the degree of joint extension will be measured with a goniometer and kept identical during the postmeasurements and follow-up measurements. During the assessment, the foot will be extended off the edge of the examination table, completely relaxed and the ankle joint will be in its resting position. Either single or multiple sweeps (depending on the size of the muscle) will be taken by moving the probe across the calf while the position of the four markers attached to the probe will be tracked by a position sensor system. A consistent, minimal pressure will be applied with the probe to avoid compression of the muscle, aided by the application of a transmission gel to improve acoustic coupling. In order to keep the pressure minimal and avoid dark 'gaps' of the lateral edges of the image ( probe width $=6 \mathrm{~cm}$ ), a customised curved gel pad will be held in between the skin and the probe's imaging surface. The probe will be kept perpendicular to the surface curvature of the shank (ie, muscle). A reconstruction of volume, anatomical cross-sectional area and length measurements will be performed using the Stradwin software (figure 2). Anatomical crosssectional area will be measured from muscle regions corresponding to each muscle's maximum anatomical crosssectional area. The location of the maximum ACSA for normally developed adults has been reported to be at $67 \%, 81 \%$ and $84 \%$ of the shank length (measured from the tuberositas calcanei to the tibial plateau) for the soleus, medial and lateral gastrocnemius, respectively. ${ }^{72}$ The location of the maximum CSA in adolescents with CP is not known and the possibility of a region-specific hypertrophy in response to resistance training cannot be excluded. However, for the standardisation purposes, we will measure the CSA at the same locations of all participants recommended for normally developed adults, ${ }^{72}$ but will also report the location of the true maximal ACSA and its interindividual variation.

\section{Tendon cross-sectional area}

Achilles tendon cross-sectional area will be obtained using a modified silicon ultrasound gel pad (Aquaflex $2 \times 9 \mathrm{~cm}$, Parker Labs) to take three discrete ultrasound images of the Achilles tendon cross-sectional area, $\sim 40 \mathrm{~mm}$ proximally to the tendon insertion. ${ }^{23}$ The tendon perimeter will then be traced using specialist software (Esaote, Italy; figure 3), and the image with the smallest cross-sectional area will be used for further analysis.

\section{Muscle and tendon force}

Plantarflexor torque will also be obtained during passive isolated ankle rotations based on previous methods $\mathbf{s}^{73} 74$ in order to estimate medial gastrocnemius muscle force. Briefly, the ankle will be rotated at a constant angular velocity of $10^{\circ} / \mathrm{s}$ through maximum plantarflexion and dorsiflexion. To do this, the participant's available range of motion will initially be determined by manually moving the foot into dorsiflexion and plantarflexion. Ultrasonography will be used to track movement of the muscle-tendon junction of the medial gastrocnemius muscle throughout the passive stretch. Achilles tendon moment arm will then be obtained using the tendon excursion method. ${ }^{21}$ The moment arm, defined as the shortest perpendicular distance between the joint centre of rotation and the line of the Achilles tendon force, will be determined by fitting a polynomial function between tendon displacement and ankle rotation data obtained from the isokinetic dynamometer. ${ }^{21}$ The moment arm will then be calculated using the formula below:

$$
\mathrm{r}=\Delta \mathrm{d} / \Delta \theta
$$

where $\mathrm{r}$ is the Achilles tendon moment arm (m), $\Delta \mathrm{d}$ represents tendon excursion $(\mathrm{m})$ and $\Delta \theta$ is the change in joint angle (rad). 
Figure 2 Three-dimensional freehand ultrasound method for the assessment of muscle volume and ACSA. A segmented and rendered medial gastrocnemius volume of a 12-year boy with a CP, GMFCS level II, moderate muscle atrophy. Three parallel transverse sections of the reconstructed muscle volume are depicted. The same transverse sections (2D B-mode images) with outlined ACSA of MG (white dotted line) are shown from proximal and distal regions of the muscle, respectively. The B-mode scans were acquired with a modified curved gel pad with a probe length of $60 \mathrm{~mm}$ and with a single sweep. The B-mode slice thickness for the 3D muscle model reconstruction was set to $5 \mathrm{~mm}$. 3D, three-dimensional; MG, medial gastrocnemius.

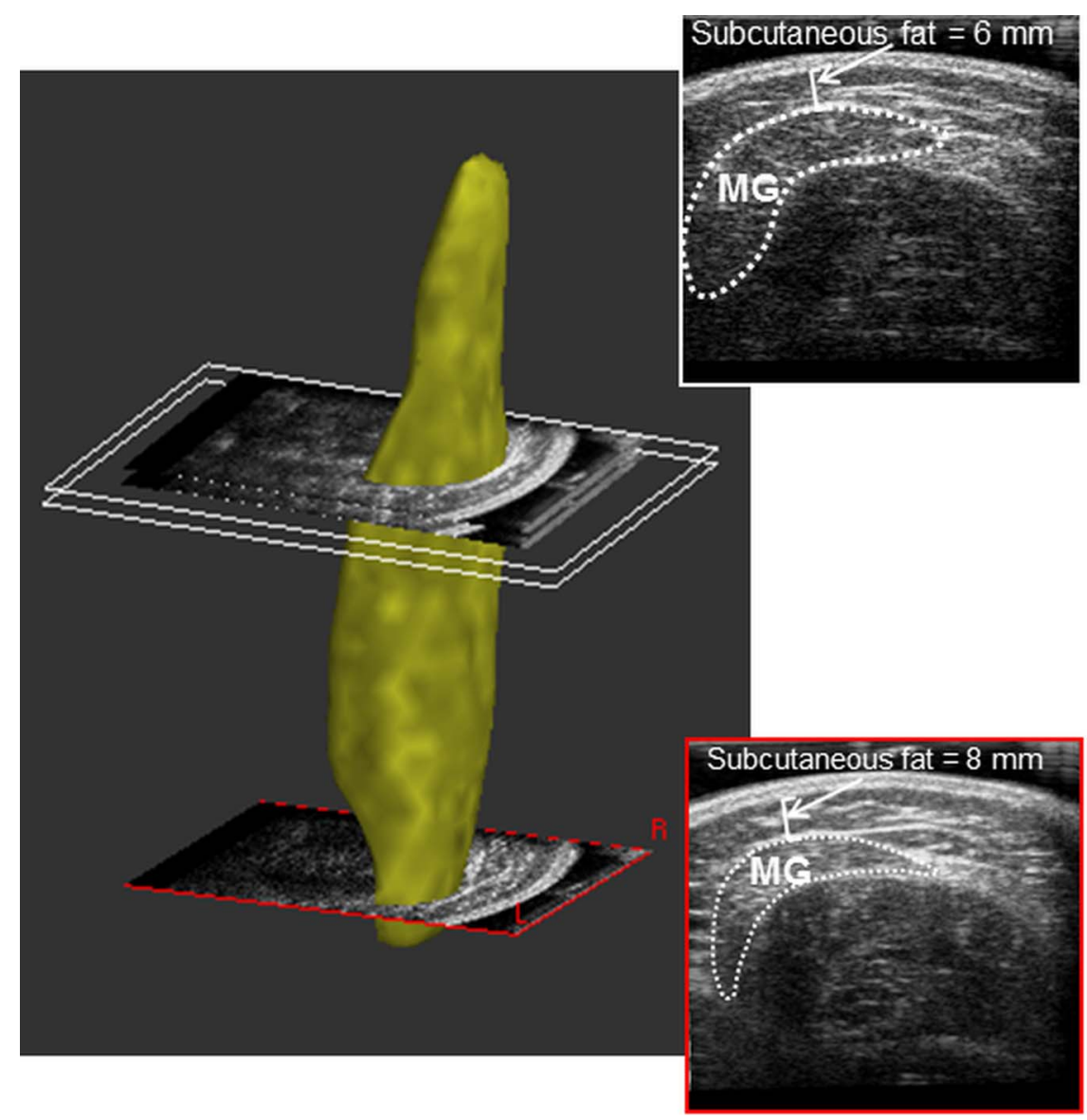

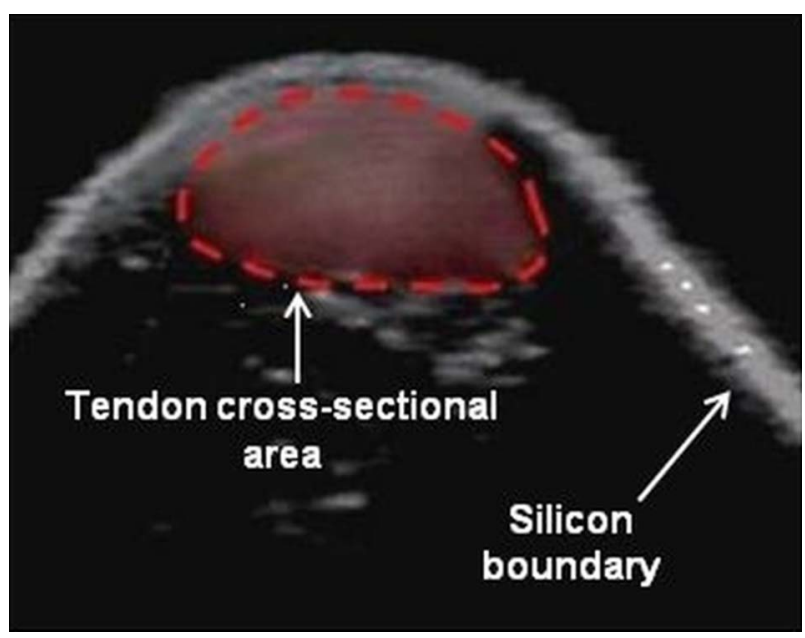

Figure 3 Measurement of Achilles tendon cross-sectional area. Area outlined in red represents the cross-section of the Achilles tendon. The silicon boundary shown on the image is formed by the silicon gel pad that is placed in between the ultrasound probe and the skin surface.

Maximal plantarflexor torque measured during maximal voluntary contractions will be divided by Achilles tendon moment arm to give an estimate of Achilles tendon force using the equation below:

$$
\mathrm{F}_{\mathrm{T}}=\mathrm{T} / \mathrm{r}
$$

where $\mathrm{F}_{\mathrm{T}}$ is the tendon force and $\mathrm{T}$ is the ankle torque as measured from the isokinetic dynamometer and corrected for antagonist activation torque as described above.

Since muscle force cannot be measured in vivo, the contribution of the medial gastrocnemius muscle to passive ankle joint torque will be estimated. To do this, passive torque will be measured during the passive rotations, and divided by Achilles tendon moment arm (as described above) to give an estimate of total muscle force. The force contribution from the medial gastrocnemius muscle will then be estimated using the formula below:

$$
\mathrm{F}_{\mathrm{MG}}=\mathrm{S}_{\mathrm{MG}} \times \mathrm{F}_{\mathrm{Total}}
$$

where $F_{M G}$ is the medial gastrocnemius muscle force $(\mathrm{N}), \mathrm{F}_{\text {Total }}$ is total muscle force and $\mathrm{S}_{\mathrm{MG}}$ is a scaling factor representing the medial gastrocnemius' contribution to the total muscle force. This scaling factor will be based on the medial gastrocnemius' anatomical crosssectional area relative to the total triceps surae crosssectional area.

\section{Muscle and tendon length}

The lengths of the medial gastrocnemius muscle and Achilles tendon will be measured, using a combination of ultrasound (Echoblaster 128, Telemed, Vilnius, Lithuania) 
and motion analysis data (Motion Analysis, Santa Rosa, California, USA). For this purpose, reflective markers will be placed on the medial epicondyle of the femur, the Achilles tendon insertion and on the ultrasound probe. Ultrasound images will be manually digitised to identify the medial gastrocnemius muscle-tendon junction in each video frame (V.9, Vicon, Oxford, UK). The position of the muscle-tendon junction in the global coordinate system of the motion analysis system will then be calculated by combining coordinates from the ultrasound probe, with local muscle-tendon junction coordinates from the ultrasound image. Using custom written analysis software (Matlab, MathWorks, Natick, Massachusetts, USA), the medial gastrocnemius muscle length will be calculated as the linear distance from the medial epicondyle marker to the global muscle-tendon junction position. Similarly, the Achilles tendon length will be calculated as the linear distance between the muscle-tendon junction and the calcaneal insertion (figure 4). The slack lengths of muscle and tendon will be calculated as the lengths at which there is a sustained increase in ankle torque above zero (baseline +2 SDs), ${ }^{34} 75$ and thus, where slack has been taken up.

\section{Muscle and tendon stiffness}

Achilles tendon stiffness will be calculated as the change in Achilles tendon force (measured from maximal voluntary contraction manoeuvres), divided by the

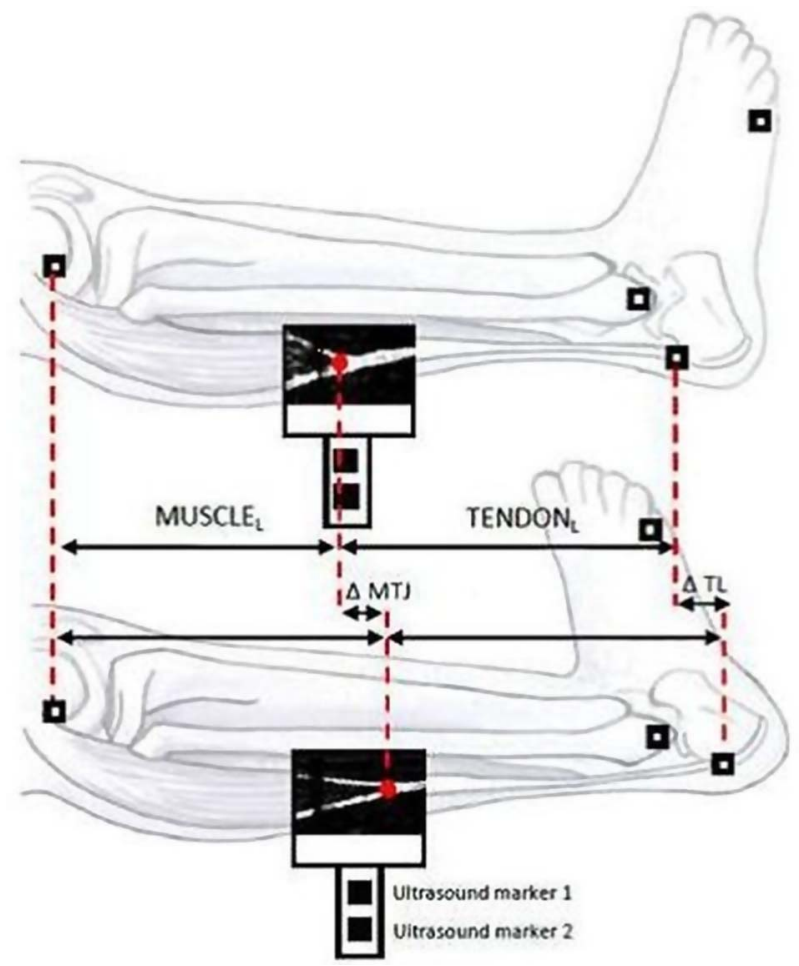

Figure 4 Experimental setup for measuring muscle length and TL. Achilles tendon length is measured as the distance from the MTJ to the calcaneus, and gastrocnemius muscle length as the distance from the mid-point of the femoral epicondyles to the MTJ. MTJ, muscle-tendon junction; TL, tendon length. corresponding change in Achilles tendon length in the linear portion of the force-length relationship (figure 5):

$$
\mathrm{k}_{\mathrm{T}}=\Delta \mathrm{F}_{\mathrm{T}} / \Delta \mathrm{L}_{\mathrm{T}}
$$

where $\mathrm{k}_{\mathrm{T}}$ is tendon stiffness, $\Delta \mathrm{F}_{\mathrm{T}}$ is change in tendon force and $\Delta \mathrm{L}_{\mathrm{T}}$ is change in tendon length. An estimate of medial gastrocnemius muscle stiffness will also be derived from the data by dividing the medial gastrocnemius force (measured from passive rotations manoeuvres) by elongation of the medial gastrocnemius muscle:

$$
\mathrm{k}_{\mathrm{MG}}=\Delta \mathrm{F}_{\mathrm{MG}} / \Delta \mathrm{L}_{\mathrm{MG}}
$$

where $k_{M G}$ is medial gastrocnemius muscle stiffness, $\Delta \mathrm{F}_{\mathrm{MG}}$ is change in medial gastrocnemius force; $\Delta \mathrm{L}_{\mathrm{MG}}$ is change in medial gastrocnemius length.

\section{Young's modulus}

The tendon's Young's modulus will be calculated by multiplying tendon stiffness by tendon resting length and dividing by tendon cross-sectional area:

Young/s modulus

$$
=\frac{\text { tendon stiffness } \times \text { tendon resting length }}{\text { tendon cross }- \text { sectional area }}
$$

\section{Muscle, tendon and fascicle strain}

Medial gastrocnemius muscle and Achilles tendon strains will be calculated by dividing muscle and tendon displacement during the passive ankle rotations, by the corresponding resting lengths. Similarly, muscle fascicle strain will be calculated by dividing muscle fascicle displacement during the passive ankle rotations by muscle fascicle length at rest. The ultrasound probe will be placed over the muscle belly revealing fascicles lying between deep and superficial aponeuroses of the medial gastrocnemius muscle. We will track the elongation of three muscle fascicles during the passive dorsiflexion trials using open source digital measurement software (Image J, National Institutes of Health (NIH)). Fascicle length will be estimated as the distance between the insertions on the deep and superficial aponeuroses. Length measurements will be averaged across the three fascicles, which will be used for subsequent analyses.

\section{Treadmill walking}

Participants will be asked to walk on a fully instrumented treadmill (Bertec Corporation, Columbus, Ohio, USA) at their preferred walking speed as previously described. ${ }^{76}$ Prior to testing, participants will perform a familiarisation session to ensure they are comfortable walking on the treadmill, and to establish a comfortable preferred walking speed. During the walking trials, participants will be barefoot and instructed to hold onto the handrail to maintain balance if required. Participants 
Figure 5 Ultrasound images

visualising the displacement of the muscle-tendon junction during an isometric muscle contraction at rest $(A)$, mid contraction (B) and at maximal force (C). Muscle-tendon junction displacement is plotted against the corresponding force to produce a force-elongation graph. Tendon stiffness is measured as the slope of the force-elongation curve.

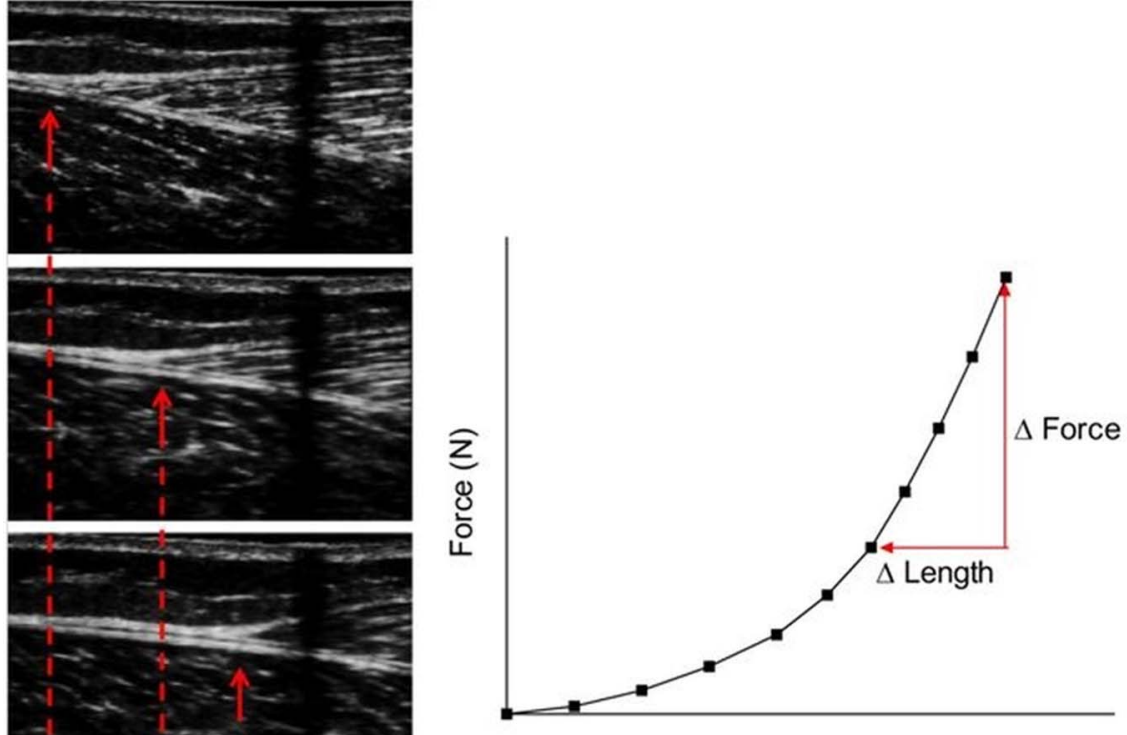

Elongation (mm)

A B C

will begin by walking at a relatively slow speed and treadmill speed will slowly increase until the participant reports the current speed is faster than their preferred speed. The treadmill speed will then be slowly decreased until the participant reports the current speed to be slower than preferred. This procedure will be repeated three times and the average of the three 'faster' and three 'slower' than preferred speeds will be taken as the participants preferred walking speed. ${ }^{76}$ This procedure also allows for treadmill familiarisation and participant warm-up. Following a mandatory 2 min rest period after familiarisation, participants will be asked to walk on the treadmill at their preferred walking speed for $2 \mathrm{~min}$ to ensure an adequate number of gait cycles are collected. ${ }^{77}$

During the walking trials, an ultrasound probe will be placed over the muscle-tendon junction of the medial gastrocnemius muscle and Achilles tendon. Three-dimensional motion analysis and ultrasound data will be combined to quantify muscle and tendon length changes as described above (see 'Muscle-tendon length' section). A second ultrasound probe will be placed over the muscle belly of the medial gastrocnemius to visualise movement of the muscle fascicles.

\section{Gait kinematics}

Knee, ankle and hip joint kinematics will be measured from a full body motion analysis marker set. For this purpose, reflective markers will be placed on the forehead and proximal sternum and then bilaterally at the distal end of each clavicle, anterior superior iliac spine, sacrum, the greater trochanters, mid-thigh, medial and lateral femoral epicondyles, on each tibia (midway between the ankle and knee), the medial and lateral malleoli, the heads of the first and fifth metatarsals and the calcaneus. The change in ankle and knee flexion angles during the stance phase will be used as the primary indicator of changes in gait kinematics.

\section{Gross motor function}

Participants' gross motor function will be assessed using dimensions D and $\mathrm{E}$ of the Gross Motor Function Measure (GMFM-66) and self-selected walking speed.

The GMFM-66 is a valid, reliable and responsive measure of change in gross motor function over time in children with CP. ${ }^{78}$ Dimension D evaluates activities in standing. Dimension E evaluates activities in walking, running and jumping. Participants' performance of dimensions D and E of the GMFM-66 will be video recorded and scored by a physiotherapist blinded to group allocation. The scores recorded for each dimension will be expressed as a percentage of the total possible score. A higher score indicates better gross motor function.

Participants' self-selected walking speed will be calculated using the time they take to walk $10 \mathrm{~m}$ over ground measured by a blinded assessor. Participants will walk $\sim 2 \mathrm{~m}$ up to the start point of a $10 \mathrm{~m}$ walkway and continue to walk for $\sim 2 \mathrm{~m}$ after the stop point of the $10 \mathrm{~m}$ walkway. Participants will not be told where the start and stop points are. Participants will complete this three times and the average speed of the three trials will be used in analysis.

\section{Process evaluation}

A mixed-methods process evaluation will be conducted in order to assess the feasibility and acceptability of the intervention. Fidelity to the intervention delivery and uptake will be assessed to identify explanatory factors relating to potential discrepancies between the expected 
and observed outcome. Data will be gathered from various sources.

Attendance and compliance to the intervention will be monitored by the physiotherapist delivering the supervised session. During each session, the physiotherapist will record details of the warm-up, cool-down and stretches, the type of exercises that were completed, the number of repetitions and sets of each exercise that were completed, the type of resistance and load that was added to each exercise, how each exercise was progressed, and details of the home exercise(s) prescribed. In order to monitor compliance with the home exercise programme and adverse events, participants will record their adherence to the home exercise programme and any potentially related or unrelated adverse events during home sessions using the patient support tool or paper exercise diary. Information from the patient support tool will be transmitted directly to the research team.

Fidelity of the resistance training programme to trial protocol will be quantified by observations of four sessions (two home sessions and two group sessions) for each participant during the 10 -week period using a protocol checklist. A member of the research team will record the quantity and quality of exercises. The number of repetitions and sets performed of each exercise, and the amount of resistance added to each exercise will be recorded. It will be noted if the exercise was progressed based on individual strength capacity. The calf muscle will be palpated during each exercise to determine if it is being activated. Aspects of the quality of the exercise (ankle range of motion that the exercise is performed through, performance of prescribed repetitions to fatigue, movement quality and degree of knee extension maintained during the exercise) will be rated on a fourpoint scale, where a higher score indicates better quality.

To determine the feasibility and acceptability of the intervention, semistructured exit interviews will be conducted with all participants in the resistance training group within 1 week of completion of the intervention. Participants will be asked to reflect on their experience, including any positive or challenging aspects of taking part in the programme, to help identify factors which may impact on adherence. Semistructured interviews will also be conducted with physiotherapists at the end of their involvement in the study to explore their experiences of delivering the resistance training programme and identify facilitators and challenges to the delivery of the intervention per protocol and potential implementation into future practice. Indicative topic guides for the semistructured interviews with adolescents who participate in physiotherapists who deliver the progressive resistance training programme are available in online supplementary files 5 and 6 , respectively.

Semistructured interviews will be transcribed verbatim, and checked against the original recordings. Data will be analysed using a multistage process (familiarisation, indexing and sorting, developing an initial framework, applying the framework, charting the data, interpretation) described in the framework approach. ${ }^{80}{ }^{81}$ Key issues arising from the data in relation to study aims and the literature will develop the a priori thematic framework both deductively and inductively. This framework will then be applied to the text prior to charting into the framework matrix and interpretation of data. Associations and differences in the data will be sought to enhance the understanding of the participants' experience of taking part in and physiotherapists' experiences of delivering resistance training.

\section{Data analysis}

To evaluate the effect of resistance training of the ankle plantarflexors on gait efficiency, activity and participation (aim 1), and muscle-tendon mechanics (aim 2) among adolescents with CP, general linear models will be used to evaluate differences in continuous data between the resistance training and usual care control group at 10 and 22 weeks, respectively. The principal analysis will be on an intention-to-treat basis. Subgroup analysis will be conducted according to: (1) the participants' ability to plantarflex the ankle, that is, rated as 'normal', 'impaired' and 'no active movement'; (2) adherence categorised as $\geq 70 \%$ or $<70 \%$ of the total dose (30 sessions). Statistical analyses will be conducted using IBM SPSS Statistics for Windows (V.22.0, IBM Corp, Amonk, New York, USA). Statistical significance will be set at $\alpha=0.05$.

\section{Sample size}

The sample size calculation was based on being able to show a minimal detectable difference in the primary outcome, NNcost, between the two groups. The calculation was based on an analysis of covariance approach, whereby the NNcost values at follow-up are considered the outcome variable, and the NNcost values at baseline are used as a covariate. Both the minimal detectable difference of 0.17 and SD of 0.31 were based on values from the literature. ${ }^{60} \mathrm{~A}$ correlation of 0.70 between baseline and follow-up NNcost values was assumed. Using a $5 \%$ significance level and $80 \%$ power, it is calculated that a sample size of 27 participants in each group is required. It is estimated that there will be a drop-out of $10 \%$, and thus it is proposed to recruit 30 people per group into the study, 60 people in total.

\section{ETHICS AND DISSEMINATION}

The trial is sponsored by Brunel University London. A parent or legal guardian of participants under 16 years and participants over 16 years will provide written informed consent. Participants under 16 years will provide written or verbal assent. All participants will take part on a voluntary basis and will be informed that they can withdraw at any time without this influencing their subsequent treatment. The trial will comply with the latest Declaration of Helsinki. Data will be kept secure 
and confidential in line with the data management plan (doi: 10.17633/rd.brunel.3485234). Pseudonymised quantitative participant data will made available in a public repository following publication of data.

The Trial Steering Committee (TSC) will monitor and supervise the progress of the trial towards its interim and overall objectives. The membership of the TSC consists of an independent Chair person, who is not involved directly with the study, a representative from the funder of the study, two independent expert members and the chief investigator. All adverse events will be reported to the TSC. Serious adverse events will be reported to the trial sponsor within 24 hours of a member of the research team becoming aware of it. Unexpected, related serious adverse events will be reported to the Research Ethics Committee within 15 days of a member of the research team becoming aware of the event.

The findings of the trial will be distributed widely to academic and non-academic audiences. The results will be published in academic journals and presented at national and international conferences. Summaries of the results will also be developed and distributed to adolescents with CP, their families and healthcare professionals through the media, professional groups and charitable organisations. The research team will work with the TSC and the STAR advisory group, which consists of young people with and without CP, to ensure that these summaries are accessible to the target audience and that the results are disseminated through appropriate channels.

\section{Author affiliations \\ ${ }^{1}$ College of Health and Life Sciences, Brunel University London, London, UK \\ ${ }^{2}$ School of Sport, Health and Applied Science, St. Mary's University, \\ Twickenham, UK \\ ${ }^{3}$ Department of Physical Therapy, University of British Columbia, Vancouver, British Columbia, Canada}

${ }^{4}$ One Small Step Gait Laboratory, Guy's Hospital, Guy's and St. Thomas' NHS Foundation Trust, London, UK

${ }^{5}$ Department of Physiotherapy, Royal Free London NHS Foundation Trust, Swiss Cottage School Development and Research Centre, London, UK

Contributors JMR and NT drafted the protocol and coordinated all subsequent revisions. JMR is the chief investigator for the trial. All authors were involved in the design of the study and the protocol development. All authors have read and approved the final draft of the protocol.

Funding Action Medical Research and the Chartered Society of Physiotherapy Charitable Trust have jointly funded this project, and it is supported by a generous grant from The Henry Smith Charity (GN2340).

Competing interests None declared.

Ethics approval This registered trial (ISRCTN90378161) has ethical approval from Brunel University London's Department of Clinical Sciences' Research Ethics Committee and the National Research Ethics Service (NRES) Committee London-Surrey Borders.

Provenance and peer review Not commissioned; externally peer reviewed.

Open Access This is an Open Access article distributed in accordance with the Creative Commons Attribution Non Commercial (CC BY-NC 4.0) license, which permits others to distribute, remix, adapt, build upon this work noncommercially, and license their derivative works on different terms, provided the original work is properly cited and the use is non-commercial. See: http:// creativecommons.org/licenses/by-nc/4.0/

\section{REFERENCES}

1. Strauss D, Brooks J, Rosenbloom L, et al. Life expectancy in cerebral palsy. An update. Dev Med Child Neurol 2008;50:487-93.

2. Day SM, Wu YW, Strauss DJ, et al. Change in ambulatory ability of adolescents and young adults with cerebral palsy. Dev Med Child Neurol 2007;49:647-53.

3. Opheim A, Jahnsen R, Olsson E, et al. Walking function, pain, and fatigue in adults with cerebral palsy: a 7-year follow-up study. Dev Med Child Neurol 2009;51:381-8.

4. Bottos M, Feliciangeli A, Sciuto L, et al. Functional status of adults with cerebral palsy and implications for treatment of children. Dev Med Child Neurol 2001;43:516-28.

5. Ryan JM, Crowley VE, Hensey O, et al. Habitual physical activity and cardiometabolic risk factors in adults with cerebral palsy. Res Dev Disabil 2014;35:1995-2002.

6. Nieuwenhuijsen C, van der Slot WM, Beelen A, et al. Inactive lifestyle in adults with bilateral spastic cerebral palsy. J Rehabil Med 2009;41:375-81.

7. Strauss D, Cable W, Shavelle R. Causes of excess mortality in cerebral palsy. Dev Med Child Neurol 1999;41:580-5.

8. Ryan JM, Hensey O, McLoughlin B, et al. Reduced moderate-to-vigorous physical activity and increased sedentary behavior is associated with elevated blood pressure values in children with cerebral palsy. Phys Ther 2014;94:1143-53.

9. Peterson MD, Ryan J, Hurvitz EA, et al. Chronic conditions in adults with cerebral palsy. JAMA 2015;314:2303-5.

10. Waters RL, Mulroy S. The energy expenditure of normal and pathologic gait. Gait Posture 1999;9:207-31.

11. Unnithan VB, Clifford C, Bar-Or O. Evaluation by exercise testing of the child with cerebral palsy. Sports Med 1998;26:239-51.

12. Brehm MA, Becher J, Harlaar J. Reproducibility evaluation of gross and net walking efficiency in children with cerebral palsy. Dev Med Child Neurol 2007;49:45-8.

13. Dallmeijer AJ, Brehm MA. Physical strain of comfortable walking in children with mild cerebral palsy. Disabil Rehabil 2011;33:1351-7.

14. Maltais DB, Pierrynowski MR, Galea VA, et al. Physical activity leve is associated with the $\mathrm{O} 2$ cost of walking in cerebral palsy. Med Sci Sports Exerc 2005;37:347-53.

15. Kerr C, Parkes J, Stevenson M, et al. Energy efficiency in gait, activity, participation, and health status in children with cerebral palsy. Dev Med Child Neurol 2008;50:204-10.

16. Kerr C, McDowell BC, Parkes J, et al. Age-related changes in energy efficiency of gait, activity, and participation in children with cerebral palsy. Dev Med Child Neurol 2011;53:61-7.

17. Unnithan VB, Dowling JJ, Frost G, et al. Cocontraction and phasic activity during GAIT in children with cerebral palsy. Electromyogr Clin Neurophysiol 1996;36:487-94.

18. Stackhouse SK, Binder-Macleod SA, Lee SC. Voluntary muscle activation, contractile properties, and fatigability in children with and without cerebral palsy. Muscle Nerve 2005;31:594-601.

19. Tedroff K, Knutson LM, Soderberg GL. Co-activity during maximum voluntary contraction: a study of four lower-extremity muscles in children with and without cerebral palsy. Dev Med Child Neurol 2008;50:377-81.

20. Unnithan VB, Dowling JJ, Frost G, et al. Role of cocontraction in the $\mathrm{O} 2$ cost of walking in children with cerebral palsy. Med Sci Sports Exerc 1996;28:1498-504.

21. Ozmun JC, Mikesky AE, Surburg PR. Neuromuscular adaptations following prepubescent strength training. Med Sci Sports Exerc 1994:26:510-14.

22. Ramsay JA, Blimkie CJ, Smith $\mathrm{K}$, et al. Strength training effects in prepubescent boys. Med Sci Sports Exerc 1990;22:605-14.

23. Hakkinen K, Komi PV. Electromyographic changes during strength training and detraining. Med Sci Sports Exerc 1983;15:455-60.

24. Reid S, Hamer P, Alderson J, et al. Neuromuscular adaptations to eccentric strength training in children and adolescents with cerebral palsy. Dev Med Child Neurol 2010;52:358-63.

25. Cronin NJ, Peltonen J, Sinkjaer T, et al. Neural compensation within the human triceps surae during prolonged walking. J Neurophysiol 2011;105:548-53.

26. Farris DJ, Sawicki GS. Human medial gastrocnemius force-velocity behavior shifts with locomotion speed and gait. Proc Natl Acad Sci USA 2012;109:977-82.

27. Lichtwark GA, Bougoulias K, Wilson AM. Muscle fascicle and series elastic element length changes along the length of the human gastrocnemius during walking and running. J Biomech 2007;40:157-64

28. Ishikawa M, Komi PV, Grey MJ, et al. Muscle-tendon interaction and elastic energy usage in human walking. J Appl Physiol 2005;99:603-8.

29. Fukunaga T, Kubo K, Kawakami Y, et al. In vivo behaviour of human muscle tendon during walking. Proc Biol Sci 2001;268:229-33. 
30. Barber L, Carty C, Modenese L, et al. Lower limb muscle fascicle function during gait in children with cerebral palsy. ISB 2015, 25th Congress of the International Society of Biomechanics.

31. Kalsi G, Fry N, Shortland A. Gastrocnemius muscle-tendon interaction during walking in typically-developing adults and children, and in children with spastic cerebral palsy. J Biomech 2016. [Epub ahead of print 8 Aug 2016].

32. Ryschon TW, Fowler MD, Wysong RE, et al. Efficiency of human skeletal muscle in vivo: comparison of isometric, concentric, and eccentric muscle action. J Appl Physiol 1997;83:867-74.

33. Theis N, Mohageghi A, Korff T. Mechanical and material properties of the triceps surae muscles and Achilles tendon in children with spastic cerebral palsy and typically developing children. J Biomech 2016. [Epub ahead of print 26 Jul 2016].

34. Barber L, Barrett $R$, Lichtwark $G$. Medial gastrocnemius muscle fascicle active torque-length and Achilles tendon properties in young adults with spastic cerebral palsy. J Biomech 2012;45:2526-30.

35. Waugh CM, Korff T, Fath $\mathrm{F}$, et al. Effects of resistance training on tendon mechanical properties and rapid force production in prepubertal children. J Appl Physiol 2014;117:257-66.

36. Shortland A. Muscle deficits in cerebral palsy and early loss of mobility: can we learn something from our elders? Dev Med Child Neurol 2009;51(Suppl 4):59-63.

37. Artero EG, Lee DC, Lavie CJ, et al. Effects of muscular strength on cardiovascular risk factors and prognosis. J Cardiopulm Rehabil Prev 2012;32:351-8.

38. Peterson MD, Gordon PM, Hurvitz EA. Chronic disease risk among adults with cerebral palsy: the role of premature sarcopoenia, obesity and sedentary behaviour. Obes Rev 2013;14:171-82.

39. Verschuren O, Ada L, Maltais DB, et al. Muscle strengthening in children and adolescents with spastic cerebral palsy: considerations for future resistance training protocols. Phys Ther 2011;91:1130-9.

40. Damiano DL. Progressive resistance exercise increases strength but does not improve objective measures of mobility in young people with cerebral palsy. J Physiother 2014;60:58.

41. Scianni A, Butler JM, Ada L, et al. Muscle strengthening is not effective in children and adolescents with cerebral palsy: a systematic review. Aust J Physiother 2009;55:81-7.

42. Park EY, Kim WH. Meta-analysis of the effect of strengthening interventions in individuals with cerebral palsy. Res Dev Disabil 2014;35:239-49.

43. Taylor NF, Dodd KJ, Baker RJ, et al. Progressive resistance training and mobility-related function in young people with cerebral palsy: a randomized controlled trial. Dev Med Child Neurol 2013;55:806-12.

44. Faigenbaum AD, Kraemer WJ, Blimkie CJ, et al. Youth resistance training: updated position statement paper from the National Strength and Conditioning Association. J Strength Cond Res 2009;23(5 Suppl):S60-79.

45. Scholtes VA, Becher JG, Comuth A, et al. Effectiveness of functiona progressive resistance exercise strength training on muscle strength and mobility in children with cerebral palsy: a randomized controlled trial. Dev Med Child Neurol 2010;52:e107-13.

46. Eek MN, Beckung $\mathrm{E}$. Walking ability is related to muscle strength in children with cerebral palsy. Gait Posture 2008;28:366-71.

47. Noble JJ, Fry NR, Lewis AP, et al. Lower limb muscle volumes in bilateral spastic cerebral palsy. Brain Dev 2014;36:294-300.

48. Ross SA, Engsberg JR. Relationships between spasticity, strength, gait, and the GMFM-66 in persons with spastic diplegia cerebral palsy. Arch Phys Med Rehabil 2007;88:1114-20.

49. Ferland $\mathrm{C}$, Lepage $\mathrm{C}$, Moffet $\mathrm{H}$, et al. Relationships between lower limb muscle strength and locomotor capacity in children and adolescents with cerebral palsy who walk independently. Phys Occup Ther Pediatr 2012;32:320-32.

50. McNee AE, Gough M, Morrissey MC, et al. Increases in muscle volume after plantarflexor strength training in children with spastic cerebral palsy. Dev Med Child Neurol 2009;51:429-35.

51. Engsberg JR, Ross SA, Collins DR. Increasing ankle strength to improve gait and function in children with cerebral palsy: a pilot study. Pediatr Phys Ther 2006;18:266-5.

52. Craig P, Dieppe P, Macintyre S, et al. Medical Research Council Guidance: developing and evaluating complex interventions: the new medical research council guidance. BMJ 2008;337:a1655.

53. Palisano RJ, Rosenbaum $P$, Bartlett $D$, et al. Content validity of the expanded and revised Gross Motor Function Classification System. Dev Med Child Neurol 2008;50:744-50.

54. Fowler EG, Staudt LA, Greenberg MB, et al. Selective Control Assessment of the Lower Extremity (SCALE): development, validation, and interrater reliability of a clinical tool for patients with cerebral palsy. Dev Med Child Neurol 2009;51:607-14.
55. Contreras B. Bodyweight strength training anatomy. Champaign, IL: Human Kinetics, 2013:160-1.

56. Arampatzis A, Karamanidis K, Stafilidis S, et al. Effect of different ankle- and knee-joint positions on gastrocnemius medialis fascicle length and EMG activity during isometric plantar flexion. J Biomech 2006;39:1891-902.

57. Hébert-Losier K, Holmberg HC. Knee angle-specific MVIC for triceps surae EMG signal normalization in weight and non weight-bearing conditions. J Electromyogr Kinesiol 2013;23:916-23.

58. Schwartz $\mathrm{MH}$. Protocol changes can improve the reliability of net oxygen cost data. Gait Posture 2007;26:494-500.

59. Schwartz MH, Koop SE, Bourke JL, et al. A nondimensional normalization scheme for oxygen utilization data. Gait Posture 2006;24:14-22.

60. Thomas SS, Buckon CE, Schwartz MH, et al. Walking energy expenditure in able-bodied individuals: a comparison of common measures of energy efficiency. Gait Posture 2009;29:592-6.

61. Mitchell LE, Ziviani J, Boyd RN. Variability in measuring physical activity in children with cerebral palsy. Med Sci Sports Exerc 2014:47:194-200.

62. Trost S, Fragala-Pinkham M, Lennon N, et al. Decision trees for detection of activity intensity in youth with cerebral palsy. Med Sci Sports Exerc 2015;48:958-66.

63. World Health Organisation. International Classification of Functioning, Disability and Health: ICF. Geneva: World Health Organisation, 2001

64. Fougeyrollas $\mathrm{P}$, Noreau $\mathrm{L}$, Bergeron $\mathrm{H}$, et al. Social consequences of long term impairments and disabilities: conceptual approach and assessment of handicap. Int $J$ Rehabil Res 1998;21:127-41.

65. Calley A, Williams S, Reid S, et al. A comparison of activity, participation and quality of life in children with and without spastic diplegia cerebral palsy. Disabil Rehabil 2012;34:1306-10.

66. Dang VM, Colver A, Dickinson HO, et al. Predictors of participation of adolescents with cerebral palsy: a European multi-centre longitudinal study. Res Dev Disabil 2014;36c:551-64.

67. Rigby BJ, Hirai N, Spikes JD, et al. The mechanical properties of rat tail tendon. J Gen Physiol 1959;43:265-83.

68. Maganaris CN, Paul JP. In vivo human tendon mechanical properties. J Physiol (Lond) 1999;521(Pt 1):307-13.

69. Damiano DL, Martellotta TL, Sullivan DJ, et al. Muscle force production and functional performance in spastic cerebral palsy: relationship of co-contraction. Arch Phys Med Rehabil 2000;81:895-900.

70. Barber L, Barrett R, Lichtwark G. Validation of a freehand 3D ultrasound system for morphological measures of the medial gastrocnemius muscle. J Biomech 2009;42:1313-19.

71. Cerniglia LM, Delmonico MJ, Lindle R, et al. Effects of acute supine rest on mid-thigh cross-sectional area as measured by computed tomography. Clin Physiol Funct Imaging 2007;27:249-53.

72. Mersmann F, Bohm S, Schroll A, et al. Validation of a simplified method for muscle volume assessment. J Biomech 2014:47:1348-52.

73. Theis N, Mohagheghi AA, Korff T. Method and strain rate dependence of Achilles tendon stiffness. J Electromyogr Kinesiol 2012;22:947-53.

74. Kay AD, Husbands-Beasley J, Blazevich AJ. Effects of contractrelax, static stretching, and isometric contractions on muscle-tendon mechanics. Med Sci Sports Exerc 2015;47:2181-90.

75. Hoang PD, Herbert RD, Todd G, et al. Passive mechanical properties of human gastrocnemius muscle-tendon units, muscle fascicles and tendons in vivo. J Exp Biol 2007;210:4159-68.

76. Dingwell JB, Marin LC. Kinematic variability and local dynamic stability of upper body motions when walking at different speeds. $J$ Biomech 2006;39:444-52.

77. Galna B, Lord S, Rochester L. Is gait variability reliable in older adults and Parkinson's disease? Towards an optimal testing protocol. Gait Posture 2013;37:580-5.

78. Nordmark E, Hagglund G, Jarnlo GB. Reliability of the gross motor function measure in cerebral palsy. Scand J Rehabil Med 1997;29:25-8.

79. Russell DJ, Rosenbaum PL, Cadman DT, et al. The gross motor function measure: a means to evaluate the effects of physical therapy. Dev Med Child Neurol 1989;31:341-52.

80. Ritchie J, Lewis J. Qualitative research practice: a guide for social science students and researchers. London: Sage, 2003.

81. Gale NK, Heath G, Cameron E, et al. Using the framework method for the analysis of qualitative data in multi-disciplinary health research. BMC Med Res Methodol 2013;13:117. 